\title{
Sectionally Pseudocomplemented Posets
}

\author{
Ivan Chajda ${ }^{1} \cdot$ Helmut Länger ${ }^{1,2}$ (D) . Jan Paseka ${ }^{3}$
}

Received: 22 May 2019 / Accepted: 25 January 2021 / Published online: 10 March 2021

(C) The Author(s) 2021

\begin{abstract}
The concept of a sectionally pseudocomplemented lattice was introduced in Birkhoff (1979) as an extension of relative pseudocomplementation for not necessarily distributive lattices. The typical example of such a lattice is the non-modular lattice $\mathrm{N}_{5}$. The aim of this paper is to extend the concept of sectional pseudocomplementation from lattices to posets. At first we show that the class of sectionally pseudocomplemented lattices forms a variety of lattices which can be described by two simple identities. This variety has nice congruence properties. We summarize properties of sectionally pseudocomplemented posets and show differences to relative pseudocomplementation. We prove that every sectionally pseudocomplemented poset is completely $L$-semidistributive. We introduce the concept of congruence on these posets and show when the quotient structure becomes a poset again. Finally, we study the Dedekind-MacNeille completion of sectionally pseudocomplemented posets. We show that contrary to the case of relatively pseudocomplemented posets, this completion need not be sectionally pseudocomplemented but we present the construction of a so-called generalized ordinal sum which enables us to construct the Dedekind-MacNeille completion provided the completions of the summands are known.
\end{abstract}

Keywords Sectional pseudocomplementation · Poset · Congruence $\cdot$ Dedekind-MacNeille completion $\cdot$ Generalized ordinal sum

\section{Introduction}

The concept of relative pseudocomplemented lattices was introduced by R. P. Dilworth in [7]. The usefulness of this concept was shown in numerous papers and books, see e.g.

\footnotetext{
Support of the research of the first two authors by the Austrian Science Fund (FWF), project I 4579-N, and the Czech Science Foundation (GAČR), project 20-09869L, entitled "The many facets of orthomodularity", as well as by ÖAD, project CZ 02/2019, entitled "Function algebras and ordered structures related to logic and data fusion", and, concerning the first author, by IGA, project PřF 2020 014, is gratefully acknowledged. The third author was supported by the project "New approaches to aggregation operators in analysis and processing of data", Nr. 18-06915S, by Czech Grant Agency (GAČR).

Helmut Länger

helmut.laenger@tuwien.ac.at
}

Extended author information available on the last page of the article. 
the famous paper [1] by R. Balbes and the monograph [2] by G. Birkhoff. This concept was extended to posets recently by the first and second author and J. Kühr in [5]. Relatively pseudocomplemented lattices turn out to be distributive, a property which also holds for relatively pseudocomplemented posets (see [5]). In order to extend relative pseudocomplementation in lattices to the non-distributive case, sectional pseudocomplementation was introduced in [3], see also [6]. The aim of the present paper is to extend sectional pseudocomplementation to posets which, of course, need not be distributive.

The concept of a sectionally pseudocomplemented lattice was introduced by the first author in [3]. Recall that a lattice $(L, \vee, \wedge)$ is sectionally pseudocomplemented if for all $a, b \in L$ there exists the pseudocomplement of $a \vee b$ with respect to $b$ in $[b, 1]$, in other words, there exists a greatest element $c$ of $L$ satisfying $(a \vee b) \wedge c=b$. In this case $c$ is called the sectional pseudocomplement of $a$ with respect to $b$ and it will be denoted by $a * b$.

The aim of this paper is to extend this concept to posets.

\section{Properties of Sectionally Pseudocomplemented Posets and Lattices}

Let $(P, \leq)$ be a poset, $a, b \in P$ and $A, B \subseteq P$. Recall that

$$
\begin{aligned}
L(A) & :=\{x \in P \mid x \leq y \text { for all } y \in A\}, \\
U(A) & :=\{x \in P \mid y \leq x \text { for all } y \in A\} .
\end{aligned}
$$

Instead of $L(\{a\}), L(\{a, b\}), L(A \cup\{a\}), L(A \cup B), L(U(A))$ we simply write $L(a), L(a, b)$, $L(A, a), L(A, B), L U(A)$, respectively. Analogously we proceed in similar cases. We also put $\downarrow(A)=\{x \in P \mid x \leq y$ for some $y \in A\}$.

We start with the following definition.

Definition 2.1 A poset $\mathbf{P}=(P, \leq)$ is called sectionally pseudocomplemented if for all $a, b \in P$ there exists a greatest $c \in P$ satisfying $L(U(a, b), c)=L(b)$. This element $c$ is called the sectional pseudocomplement $a * b$ of $a$ with respect to $b$. The poset $\mathbf{P}$ is called strongly sectionally pseudocomplemented if it is sectionally pseudocomplemented, it has a greatest element 1 and it satisfies the condition $x \leq(x * y) * y$ (which, as we will see later, is equivalent to the identity $x *((x * y) * y) \approx 1)$.

In the following we list several important properties of sectionally pseudocomplemented posets.

Theorem 2.2 Let $\mathbf{P}=(P, \leq, *, 1)$ be a sectionally pseudocomplemented poset with 1 . Then the following hold:

(i) $x \leq y$ if and only if $x * y=1$,

(ii) $x * x \approx x * 1 \approx 1$,

(iii) $1 * x \approx x$,

(iv) $x *(y * x) \approx 1$,

(v) if $x * y=1$ or $y * x=1$, then $x *((x * y) * y)=1$,

(vi) if $x * y=1$, then $(y * z) *(x * z)=1$.

Proof Let $a, b, c \in P$. 
(i) The following are equivalent:

$$
\begin{aligned}
a & \leq b, \\
U(a, b) & =U(b), \\
L U(a, b) & =L(b), \\
L(U(a, b), 1) & =L(b), \\
a * b & =1 .
\end{aligned}
$$

(ii) It follows from (i).

(iii) The following are equivalent:

$$
\begin{aligned}
L(U(1, a), b) & =L(a), \\
L(b) & =L(a), \\
b & =a .
\end{aligned}
$$

(iv) We have $L(U(a, b), b)=L(b)$ implies $b \leq a * b$.

(v) If $a \leq b$, then $a \leq b=1 * b=(a * b) * b$ according to (iii) and (i), and if $b \leq a$, then $L(U(a * b, b), a)=L(a * b, a)=L(U(a, b), a * b)=L(b)$ and hence $a \leq(a * b) * b$.

(vi) If $a \leq b$, then $L(c) \subseteq L(U(a, c), b * c) \subseteq L(U(b, c), b * c)=L(c)$, i.e. $L(U(a, c), b * c)=L(c)$ whence $b * c \leq a * c$.

In a lattice $(P, \vee, \wedge)$ the equation $L(U(a, b), c)=L(b)$ is equivalent to $(a \vee b) \wedge c=b$. If $\mathbf{P}$ is a join-semilattice then $L(U(a, b), c)=L(U(a \vee b), c)=L(a \vee b, c)$.

Recall that a poset $\mathbf{P}$ with 0 is called pseudocomplemented (cf. [10]) if for every $a \in P$ there exists a greatest $b \in P$ with $L(a, b)=L(0)$. The subsets of $P$ of the form $U(x)$ $(x \in P)$ are called sections of $\mathbf{P}$. The following are equivalent:

Every section of $\mathbf{P}$ is pseudocomplemented,

for all $b \in P$ and $a \in U(b)$ there exists a greatest $c \in U(b)$ with

$$
L(a, c) \cap U(b)=L(b) \cap U(b) .
$$

(Observe that $L(b) \cap U(b)=\{b\}$.)

If $\mathbf{P}$ is a join-semilattice then we have the following analogy between the property that $\mathbf{P}$ is sectionally pseudocomplemented and the property that every section of $\mathbf{P}$ is pseudocomplemented.

The following are equivalent:

$\mathbf{P}$ is sectionally pseudocomplemented,

for all $a, b \in P$ there exists a greatest $c \in P$ with $L(a \vee b, c)=L(b)$,

for all $b \in P$ and $a \in U(b)$ there exists a greatest $c \in P$ with $L(a, c)=L(b)$,

for all $b \in P$ and $a \in U(b)$ there exists a greatest $c \in U(b)$ with $L(a, c)=L(b)$.

If our sectionally pseudocomplemented poset is even a meet-semilattice we can obtain more.

Proposition 2.3 In every sectionally pseudocomplemented meet-semilattice $\mathbf{P}=(P, \leq$, $*, 1)$ the section $U(b)$ is a pseudocomplemented poset for all $b \in P$. 
Proof Let $b \in P$ and $a \in U(b)$. Since $\mathbf{P}$ is sectionally pseudocomplemented there exists a greatest $c \in P$ satisfying $L(U(a, b), c)=L(b)$. Let us compute

$$
L(U(a, b), c)=L(U(a), c)=L(a, c)=L(b) .
$$

Therefore also $L(a, c) \cap U(b)=L(b) \cap U(b)$. Now, let $d \in P$ such that $L(a, d) \cap U(b)=$ $L(b) \cap U(b)$. Then $b \in L(a, d)$. Let us compute

$$
L(U(a, b), d)=L(U(a), d)=L(a, d)=L(a \wedge d) \supseteq L(b) .
$$

Hence $b \leq a \wedge d$. Then $a \wedge d \in L(a, d) \cap U(b)=\{b\}$ and we obtain $b=a \wedge d$. Therefore $L(U(a, b), d)=L(b)$, i.e., $d \leq c$.

The following example shows that there really exist sectionally pseudocomplemented posets which are not strongly sectionally pseudocomplemented. Hence, we cannot expect that every sectionally pseudocomplemented poset satisfies the condition $x \leq(x * y) * y$.

Example 2.4 The poset visualized in Fig. 1 is sectionally pseudocomplemented and $*$ has the operation table shown in Fig. 1, but it is not strongly sectionally pseudocomplemented since $c \not \leq a=f * a=(c * a) * a$.

In [5], the first and second author also presented a slightly different notion of sectionally pseudocomplemented poset. Hence a poset $\mathbf{P}$ introduced in [5] will be called $C K L$-sectionally pseudocomplemented if for all $a, b \in P$, there exists $d \in P$ (called a $C K L$-sectional pseudocomplement) such that for all $x \in P$

(1) $L(U(a, b), U(x, b))=L(b)$ if and only if $d \in U(x, b)$.

Further, it was shown in [5] that if such a $d$ exists then it is unique and satisfies $b \leq d$ and $L(U(a, b), d)=L(b)$. If $e \in P$ and $L(U(a, b), e)=L(b)$ then $b \leq e$ and hence $L(U(a, b), U(e, b))=L(U(a, b), U(e))=L(U(a, b), e)=L(b)$, thus, by (1), $d \in U(e, b)$ which implies $e \leq d$. This shows that $d$ is the greatest $c \in P$ satisfying $L(U(a, b), c)=L(b)$. In other words, if $d$ is the CKL-sectional pseudocomplement of $a$ with respect to $b$ then $d$ is the sectional pseudocomplement of $a$ with respect to $b$ as defined in the present paper.

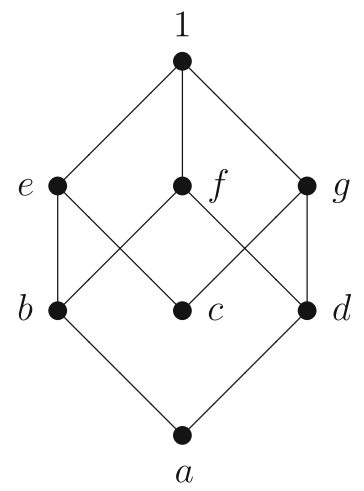

\begin{tabular}{c|llllllll}
$*$ & $a$ & $b$ & $c$ & $d$ & $e$ & $f$ & $g$ & 1 \\
\hline$a$ & 1 & 1 & $c$ & 1 & 1 & 1 & 1 & 1 \\
$b$ & $g$ & 1 & $c$ & $g$ & 1 & 1 & $g$ & 1 \\
$c$ & $f$ & $f$ & 1 & $f$ & 1 & $f$ & 1 & 1 \\
$d$ & $e$ & $e$ & $c$ & 1 & $e$ & 1 & 1 & 1 \\
$e$ & $d$ & $f$ & $c$ & $d$ & 1 & $f$ & $g$ & 1 \\
$f$ & $a$ & $e$ & $c$ & $g$ & $e$ & 1 & $g$ & 1 \\
$g$ & $b$ & $b$ & $c$ & $f$ & $e$ & $f$ & 1 & 1 \\
1 & $a$ & $b$ & $c$ & $d$ & $e$ & $f$ & $g$ & 1
\end{tabular}

Fig. 1 
Proposition 2.5 Every CKL-sectionally pseudocomplemented poset $\mathbf{P}=(P, \leq, *, 1)$ with top element 1 is strongly sectionally pseudocomplemented.

Proof From the above considerations we have that $\mathbf{P}$ is sectionally pseudocomplemented. Let us check that $x \leq(x * y) * y$ for all $x, y \in P$. By Theorem 2.2 (iv), we have $y \leq(x * y) * y$. Because $x * y$ is the sectional pseudocomplement of $x$ with respect to $y$, we can compute

$$
L(U(x * y, y), U(x, y))=L(U(x * y), U(x, y))=L(U(x, y), x * y)=L(y) .
$$

Since $\mathbf{P}$ is CKL-sectionally pseudocomplemented the CKL-sectional pseudocomplement of $x * y$ with respect to $y$ is $(x * y) * y$ and, by $(1),(x * y) * y \in U(x, y)$, i.e., $x \leq(x * y) * y$.

In particular, we have from Example 2.4 that there exists a sectionally pseudocomplemented poset which is not CKL-sectionally pseudocomplemented.

For join-semilattices we know more. We are going to show that every sectionally pseudocomplemented join-semilattice with 1 is strongly sectionally pseudocomplemented.

Theorem 2.6 Let $\mathbf{P}=(P, \leq, 1)$ be a join-semilattice with a top element 1. The following conditions are equivalent:

(i) $\mathbf{P}$ is CKL-sectionally pseudocomplemented.

(ii) $\mathbf{P}$ is strongly sectionally pseudocomplemented.

(iii) $\mathbf{P}$ is sectionally pseudocomplemented.

Proof Clearly, (i) implies (ii) and (ii) implies (iii). Let us check that (iii) implies (i). Let $a, b \in P$ and let $a * b$ be the sectional pseudocomplement of $a$ with respect to $b$. Then for all $x \in P$ the following are equivalent:

$$
\begin{aligned}
& L(U(a, b), U(x, b))=L(b), \\
& L(U(a, b), x \vee b)=L(b), \\
& x \vee b \leq a * b, \\
& a * b \in U(x, b) .
\end{aligned}
$$

Therefore $a * b$ is the CKL-sectional pseudocomplement of $a$ with respect to $b$ for all $a, b \in P$.

The following result follows from the previous theorem.

Corollary 2.7 [3] Every sectionally pseudocomplemented lattice $\mathbf{L}=(L, \vee, \wedge, *)$ satisfies $x \vee y \leq(x * y) * y$.

Recall from [5] or [8] that the relative pseudocomplement of a with respect to $b$ is the greatest $d \in P$ satisfying $L(a, d) \subseteq L(b)$. The poset $\mathbf{P}=(P, \leq)$ is called relatively pseudocomplemented if for all $a, b \in P$ the relative pseudocomplement of $a$ with respect to $b$ exists. Every relatively pseudocomplemented join-semilattice is sectionally pseudocomplemented. This can be seen as follows: Assume $\mathbf{P}$ to be a relatively pseudocomplemented join-semilattice and let $a, b \in P$. Then there exists a greatest element $d$ of $P$ with $L(a \vee b, d) \subseteq L(b)$. Since $L(a \vee b, b)=L(b)$ we have $b \leq d$ and hence $L(a \vee b, d)=L(b)$. If $e \in P$ and $L(a \vee b, e)=L(b)$ then $L(a \vee b, e) \subseteq L(b)$ and hence $e \leq d$. This shows that $d$ is the sectional pseudocomplement of $a$ with respect to $b$. 
It was shown by Y. S. Pawar [8] that the Dedekind-MacNeille completion DM(P) of a relatively pseudocomplemented poset $\mathbf{P}$ is relatively pseudocomplemented and that the relative pseudocomplementation in $\mathbf{D M}(\mathbf{P})$ extends the relative pseudocomplementation in $\mathbf{P}$ if $\mathbf{P}$ is canonically embedded into $\mathbf{D M}(\mathbf{P})$. We will use this fact to prove the following.

Theorem 2.8 Every relatively pseudocomplemented poset $\mathbf{P}$ is sectionally pseudocomplemented.

Proof Let $a, b \in P$ and let $a \circ b$ denote the relative pseudocomplement of $a$ with respect to $b$. We have to show that $a \circ b$ is the sectional pseudocomplement of $a$ with respect to $b$.

From the above note we know that $L(a \circ b)$ is the relative pseudocomplement of $L(a)$ with respect to $L(b)$ in the lattice $\mathbf{D M}(\mathbf{P})$. Hence it is also the sectional pseudocomplement of $a$ with respect to $b$ in $\mathbf{D M}(\mathbf{P})$. Since $\mathbf{D M}(\mathbf{P})$ is a lattice, $L(a \circ b)$ is the greatest element $C$ of $\mathbf{D M}(\mathbf{P})$ satisfying $(L(a) \vee L(b)) \wedge C=L(b)$. But this means exactly that $L(a \circ b)$ is the greatest element $C$ of $\mathbf{D M}(\mathbf{P})$ such that $L(U(a, b), U(C))=L(b)$. In particular, $a \circ b$ is the greatest element $c$ of $P$ such that $L(U(a, b), c)=L(U(a, b), U(c))=L(b)$.

It was shown in [3] that the class of sectionally pseudocomplemented lattices forms a variety. However, the defining identities given in [3] are rather complicated. We present some simpler identities as follows.

Theorem 2.9 The class of sectionally pseudocomplemented lattices forms a variety which besides the lattice axioms is determined by the following identities:

(i) $z \vee y \leq x *((x \vee y) \wedge(z \vee y))$,

(ii) $(x \vee y) \wedge(x * y) \approx y$.

Proof Let $\mathbf{L}=(L, \vee, \wedge)$ be a lattice and $a, b, c \in L$. First assume $\mathbf{L}$ to be sectionally pseudocomplemented. If $d:=(a \vee b) \wedge(c \vee b)$, then

$$
\begin{aligned}
a \vee d & =a \vee((a \vee b) \wedge(c \vee b))=a \vee(b \vee((a \vee b) \wedge(c \vee b)))= \\
& =(a \vee b) \vee((a \vee b) \wedge(c \vee b))=a \vee b, \\
c \vee d & =c \vee((a \vee b) \wedge(c \vee b))=c \vee(b \vee((a \vee b) \wedge(c \vee b)))= \\
& =(c \vee b) \vee((a \vee b) \wedge(c \vee b))=c \vee b
\end{aligned}
$$

and hence

$$
d \leq(a \vee d) \wedge(c \vee d)=(a \vee b) \wedge(c \vee b)=d,
$$

i.e. $(a \vee d) \wedge(c \vee d)=d$ which shows

$$
c \vee b=c \vee d \leq a * d=a *((a \vee b) \wedge(c \vee b))
$$

proving (i). Identity (ii) follows from the definition of $*$. Conversely, assume $\mathbf{L}$ to satisfy (i) and (ii). Then $(a \vee b) \wedge(a * b)=b$ according to (ii). If $(a \vee b) \wedge c=b$, then $b \leq c$ and hence $(a \vee b) \wedge(c \vee b)=(a \vee b) \wedge c=b$ whence

$$
c=c \vee b \leq a *((a \vee b) \wedge(c \vee b))=a * b
$$

according to (i). This shows that $a * b$ is the sectional pseudocomplement of $a$ with respect to $b$.

Evidently, every relatively pseudocomplemented lattice $(L, \vee, \wedge)$ is sectionally pseudocomplemented since for $a, b \in L$ we have $a * b=(a \vee b) \circ b$ where $*$ and $\circ$ denote 
sectional and relative pseudocomplementation, respectively. (Observe that ( $a \vee b) \wedge b=b$ and hence $(a \vee b) \wedge((a \vee b) \circ b)=b$. ) The following poset is an example of a sectionally pseudocomplemented poset which is neither a lattice nor relatively pseudocomplemented.

Example 2.10 The poset visualized in Fig. 2 is strongly sectionally pseudocomplemented and the operation table of $*$ is shown in Fig. 2.

Evidently, this poset is not a lattice. However, it is also not relatively pseudocomplemented since the relative pseudocomplement of $c$ with respect to $a$ does not exist. The poset visualized in Fig. 3 is a strongly sectionally pseudocomplemented lattice and $*$ has the operation table shown in Fig. 3, but this poset is not relatively pseudocomplemented since the relative pseudocomplement of $c$ with respect to $a$ does not exist.

Remark 2.11 Assertion (vi) of Theorem 2.2 says that $*$ is antitone in the first variable, i.e. $x \leq y$ implies $y * z \leq x * z$. Contrary to the case of relatively pseudocomplemented posets, sectional pseudocomplementation is not monotone in the second variable. Namely, in the poset visualized in Fig. 2 we have $0 \leq a$, but $b * 0=c \not \leq a=b * a$. However, $*$ need not be monotone in the second variable also in sectionally pseudocomplemented lattices as the following example shows.

Example 2.12 The lattice visualized in Fig. 4 is sectionally pseudocomplemented and $*$ has the operation table shown in Fig. 4. Here we have $0<a$ and $b * 0=d \| c=b * a$.

For every algebra $(A, *, 1)$ of type $(2,0)$ and every subset $B$ of $A$ put

$$
\begin{gathered}
L(B):=\{x \in A \mid x * y=1 \text { for all } y \in B\}, \\
U(B):=\{x \in A \mid y * x=1 \text { for all } y \in B\} .
\end{gathered}
$$

We are going to show that sectionally pseudocomplemented posets can be defined as certain groupoids.

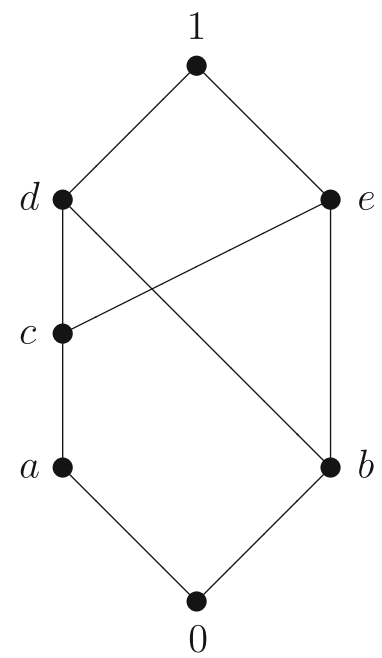

\begin{tabular}{c|ccccccc}
$*$ & 0 & $a$ & $b$ & $c$ & $d$ & $e$ & 1 \\
\hline 0 & 1 & 1 & 1 & 1 & 1 & 1 & 1 \\
$a$ & $b$ & 1 & $b$ & 1 & 1 & 1 & 1 \\
$b$ & $c$ & $a$ & 1 & $c$ & 1 & 1 & 1 \\
$c$ & $b$ & $a$ & $b$ & 1 & 1 & 1 & 1 \\
$d$ & 0 & $a$ & $b$ & $c$ & 1 & $e$ & 1 \\
$e$ & 0 & $a$ & $b$ & $c$ & $d$ & 1 & 1 \\
1 & 0 & $a$ & $b$ & $c$ & $d$ & $e$ & 1
\end{tabular}

Fig. 2 


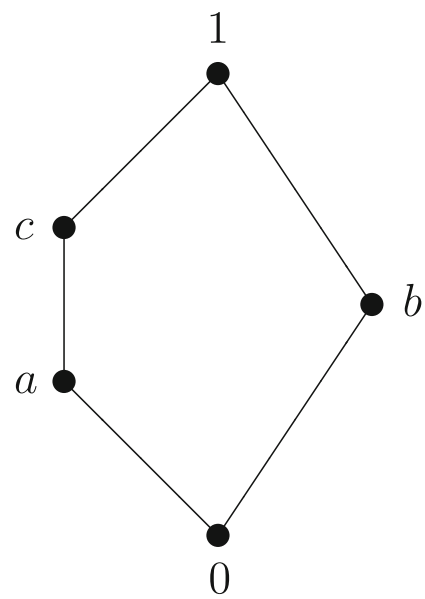

\begin{tabular}{c|ccccc}
$*$ & 0 & $a$ & $b$ & $c$ & 1 \\
\hline 0 & 1 & 1 & 1 & 1 & 1 \\
$a$ & $b$ & 1 & $b$ & 1 & 1 \\
$b$ & $c$ & $a$ & 1 & $c$ & 1 \\
$c$ & $b$ & $a$ & $b$ & 1 & 1 \\
1 & 0 & $a$ & $b$ & $c$ & 1
\end{tabular}

Fig. 3

Theorem 2.13 An algebra $(A, *, 1)$ of type $(2,0)$ can be organized into a sectionally pseudocomplemented poset with 1 if and only if the following hold:

(i) $x * x \approx 1$,

(ii) $x * y=y * x=1 \Rightarrow x=y$,

(iii) $x * y=y * z=1 \Rightarrow x * z=1$,

(iv) $L(U(x, y), x * y)=L(y)$,

(v) $L(U(x, y), z)=L(y) \Rightarrow z *(x * y)=1$.

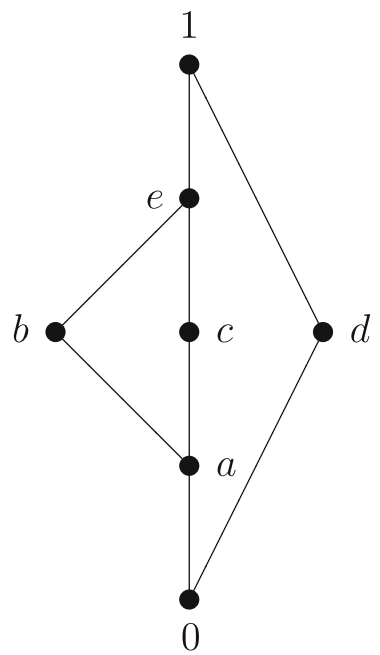

\begin{tabular}{l|lllllll}
$*$ & 0 & $a$ & $b$ & $c$ & $d$ & $e$ & 1 \\
\hline 0 & 1 & 1 & 1 & 1 & 1 & 1 & 1 \\
$a$ & $d$ & 1 & 1 & 1 & $d$ & 1 & 1 \\
$b$ & $d$ & $c$ & 1 & $c$ & $d$ & 1 & 1 \\
$c$ & $d$ & $b$ & $b$ & 1 & $d$ & 1 & 1 \\
$d$ & $e$ & $a$ & $b$ & $c$ & 1 & $e$ & 1 \\
$e$ & $d$ & $a$ & $b$ & $c$ & $d$ & 1 & 1 \\
1 & 0 & $a$ & $b$ & $c$ & $d$ & $e$ & 1
\end{tabular}

Fig. 4 
Proof The necessity of the conditions is clear. Conversely, if $\leq$ is defined on $A$ by $x \leq$ $y$ if and only if $x * y=1$, then conditions (i) - (v) imply $(A, \leq, *, 1)$ is a sectionally pseudocomplemented poset.

Recall that a lattice $(L, \vee, \wedge)$ is called completely meet-semidistributive if the following holds:

$$
\text { If } \emptyset \neq M \subseteq L, a, b \in L \text { and } x \wedge a=b \text { for all } x \in M \text {, then }(\bigvee M) \wedge a=b .
$$

For posets, we modify this concept as follows.

Definition 2.14 A poset $(P, \leq)$ is called completely L-semidistributive if the following holds:

If $\emptyset \neq M \subseteq P, a, b \in P$ and $L(x, a)=L(b)$ for all $x \in M$, then $L(U(M), a)=L(b)$.

Theorem 2.15 Let $\mathbf{P}=(P, \leq, *)$ be a sectionally pseudocomplemented poset. Then $\mathbf{P}$ is completely L-semidistributive.

Proof If $\emptyset \neq M \subseteq P, a, b \in P$ and $L(x, a)=L(b)$ for all $x \in M$, then $b \leq a$ and therefore $L(U(a, b), x)=L(a, x)=L(b)$ for all $x \in M$ and hence $x \leq a * b$ for all $x \in M$ whence $a * b \in U(M)$ which finally implies

$$
L(b) \subseteq L(U(M), a) \subseteq L(a * b, a)=L(U(a, b), a * b)=L(b),
$$

i.e. $L(U(M), a)=L(b)$.

\section{Congruences in Sectionally Pseudocomplemented Posets}

Theorem 2.2 (i) shows that in a sectionally pseudocomplemented poset $(P, \leq, *, 1)$ with 1 , $\leq$ is uniquely determined by $*$. Let $(P, \leq, *, 1)$ be a sectionally pseudocomplemented poset with 1 and $\Theta \in \operatorname{Con}(P, *)$. We are interested in the question when $\left(P / \Theta, \leq^{\prime}\right)$ is a poset where $\leq^{\prime}$ is defined by $[x] \Theta \leq^{\prime}[y] \Theta$ if $[x] \Theta *[y] \Theta=[1] \Theta(x, y \in P)$. We will see that this is the case if $\Theta$ is convex, i.e. every class of $\Theta$ is a convex subset of $(P, \leq)$.

First we show that if $(P, \leq, *, 1)$ is a finite sectionally pseudocomplemented poset with 1 , then $(P, *)$ has convex congruences.

In the following lemma and theorem we frequently use Theorem $2.2(\mathrm{v})$.

Lemma 3.1 Let $(P, \leq, *, 1)$ be a sectionally pseudocomplemented poset with 1 satisfying the Ascending Chain Condition, let $a, b \in P$ and $\Theta \in \operatorname{Con}(P, *)$ and assume $a<b<$ $(b * a) * a$ and $a \Theta(b * a) * a$. Then $a \Theta b$.

Proof Assume $(a, b) \notin \Theta$. Put $a_{1}:=a, a_{2}:=b$ and $a_{n}:=\left(a_{n-1} * a_{n-2}\right) * a_{n-2}$ for $n \geq 3$. Then $a_{1}<a_{2}<a_{3}$ and $a_{3} \Theta a_{1}$. Now

$$
a_{4}=\left(a_{3} * a_{2}\right) * a_{2} \Theta\left(a_{1} * a_{2}\right) * a_{2}=1 * a_{2}=a_{2} .
$$

Moreover, $a_{3} \leq a_{4}$. Now $a_{3}=a_{4}$ would imply $a_{1} \Theta a_{3}=a_{4} \Theta a_{2}$, a contradiction. This shows $a_{3}<a_{4}$. Now

$$
a_{5}=\left(a_{4} * a_{3}\right) * a_{3} \Theta\left(a_{2} * a_{3}\right) * a_{3}=1 * a_{3}=a_{3} .
$$


Moreover, $a_{4} \leq a_{5}$. Now $a_{4}=a_{5}$ would imply $a_{1} \Theta a_{3} \Theta a_{5}=a_{4} \Theta a_{2}$, a contradiction. This shows $a_{4}<a_{5}$. Going on in this way we would obtain an infinite strictly ascending chain $a_{1}<a_{2}<a_{3}<a_{4}<a_{5}<\cdots$ contradicting the Ascending Chain Condition. This shows $a \Theta b$.

Hence, we conclude

Theorem 3.2 Let $(P, \leq, *, 1)$ be a sectionally pseudocomplemented poset with 1 satisfying the Ascending Chain Condition and let $\Theta \in \operatorname{Con}(P, *)$. Then $\Theta$ is convex.

Proof Assume $a, b, c \in P, a<b<c$ and $(a, c) \in \Theta$. Then $b \leq(b * a) * a$. If $b=(b * a) * a$, then $b=(b * a) * a \Theta(b * c) * a=1 * a=a$. If $b<(b * a) * a$, then $a \Theta b$ according to Lemma 3.1.

Corollary 3.3 If $(P, \leq, *, 1)$ is a finite sectionally pseudocomplemented poset with 1 , then $(P, *)$ has convex congruences.

For the infinite case we have the following result.

Theorem 3.4 Let $(P, \leq, *, 1)$ be a sectionally pseudocomplemented poset with 1 such that $x, y \in P, x<y<1, x \nprec y$ and $x<y * x$ together imply $\Theta(x, y)=P^{2}$. Then $(P, *)$ has convex congruences.

Proof Let $\Theta \in \operatorname{Con}(P, *)$ and $a, b, c \in P$ and assume $a<b<c$ and $(a, c) \in \Theta$. If $c=1$, then

$$
a \Theta 1=a * b \Theta 1 * b=b .
$$

If $c<1$ and $a<c * a$, then $\Theta(a, c)=P^{2}$ and hence $\Theta=P^{2}$ which implies $a \Theta b$. If $c<1$ and $a=c * a$, then

$a=1 * a=(a * a) * a \Theta(c * a) * a=a * a=1=a * b=(c * a) * b \Theta(a * a) * b=1 * b=b$.

This shows that $\Theta$ is convex.

Let $(P, \leq, *, 1)$ be a sectionally pseudocomplemented poset with 1 and $\Theta \in \operatorname{Con}(P, *)$. We define a binary relation $\leq^{\prime}$ on $P / \Theta$ by $[x] \Theta \leq^{\prime}[y] \Theta$ if $[x] \Theta *[y] \Theta=[1] \Theta(x, y \in P)$. Now we can prove

Theorem 3.5 Let $(P, \leq, *, 1)$ be a strongly sectionally pseudocomplemented poset and let $a, b \in P$ and $\Theta$ a convex congruence on $(A, *)$. Then the following hold:

(i) If $[a] \Theta \leq^{\prime}[b] \Theta$, then there exists some $d \in[b] \Theta$ with $a \leq d$,

(ii) if $a \leq b$, then $[a] \Theta \leq^{\prime}[b] \Theta$,

(iii) $\left(P / \Theta, \leq^{\prime}\right)$ is a poset.

\section{Proof}

(i) Put $d:=(a * b) * b$. Then $d=(a * b) * b \Theta 1 * b=b$ and $a \leq(a * b) * b=d$.

(ii) If $a \leq b$, then $a * b=1$ according to Theorem 2.2 and hence $a * b \Theta 1$, i.e. $[a] \Theta \leq^{\prime}[b] \Theta$. 
(iii) Obviously, $\leq^{\prime}$ is reflexive. Now assume $[a] \Theta \leq^{\prime}[b] \Theta \leq^{\prime}[a] \Theta$. Then, by (i), there exists some $d \in[b] \Theta$ with $a \leq d$. Because of $[d] \Theta=[b] \Theta \leq^{\prime}[a] \Theta$ there exists some $e \in[a] \Theta$ with $d \leq e$. Since $a \leq d \leq e,(a, e) \in \Theta$ and $\Theta$ is convex we conclude $(a, d) \in \Theta$. Therefore $[a] \Theta=[d] \Theta=[b] \Theta$ which proves antisymmetry of $\leq^{\prime}$. Finally, let $c \in P$ and assume $[a] \Theta \leq^{\prime}[b] \Theta \leq^{\prime}[c] \Theta$. Then, by (i) there exists some $f \in[b] \Theta$ with $a \leq f$ and because of $[f] \Theta=[b] \Theta \leq^{\prime}[c] \Theta$ some $g \in[c] \Theta$ with $f \leq g$. Because of $a \leq f \leq g$ we have $a \leq g$ which implies $[a] \Theta \leq^{\prime}[g] \Theta=[c] \Theta$ by (ii), proving transitivity of $\leq$ '.

Lemma 3.6 Let $\mathbf{P}=(P, \leq, *, 1)$ be a strongly sectionally pseudocomplemented poset, $a \in P$ and $\Theta \in \operatorname{Con}(P, *)$. Then $[a] \Theta$ is up-directed. Hence, if $\mathbf{P}$ satisfies the Ascending Chain Condition, then $[a] \Theta$ has a greatest element.

Proof If $b, c \in[a] \Theta$, then

$$
(b * c) * c \in[(c * c) * c] \Theta=[1 * c] \Theta=[c] \Theta=[a] \Theta,
$$

$b \leq(b * c) * c$ since $\mathbf{P}$ is strongly sectionally pseudocomplemented, and $c \leq(b * c) * c$ according to Theorem 2.2 (iv).

From Theorem 3.5 we have: If $(P, \leq, *, 1)$ is a strongly sectionally pseudocomplemented poset, $\Theta$ a convex congruence on $(P, *), a$ the greatest element of $[a] \Theta$ and $b$ the greatest element of $[b] \Theta$, then $a \leq b$ if and only if $[a] \Theta \leq^{\prime}[b] \Theta$.

Now we solve the problem for which $\Theta \in \operatorname{Con}(P, *)$ the quotient $P / \Theta$ is again sectionally pseudocomplemented. We can state a sufficient condition.

Definition 3.7 Let $\mathbf{P}=(P, \leq, *)$ be a sectionally pseudocomplemented poset and $\Theta \in$ $\operatorname{Con}(P, *)$. We say that $\Theta$ is strong if the following holds: If $a, b \in P, a$ is the greatest element of $[a] \Theta$ and $b$ the greatest element of $[b] \Theta$, then $a * b$ is the greatest element of $[a * b] \Theta$. In this case we define $[a] \Theta *^{\prime}[b] \Theta:=[a * b] \Theta$.

It is easy to see that if $\Theta$ is strong, $a, b, c, d \in P, a \leq b$ and $c$ is the greatest element of $[a] \Theta$ and $d$ the greatest element of $[b] \Theta$, then $c \leq d$.

Theorem 3.8 Let $\mathbf{P}=(P, \leq, *, 1)$ be a strongly sectionally pseudocomplemented poset and $\Theta \in \operatorname{Con}(P, *)$ a strong congruence. If $\mathbf{P}$ satisfies the Ascending Chain Condition, then $\left(P / \Theta, \leq^{\prime}, *^{\prime},[1] \Theta\right)$ is a strongly sectionally pseudocomplemented poset.

Proof Since $\mathbf{P}$ satisfies the Ascending Chain Condition we know from Theorems 3.2 and 3.5 that $\Theta$ is convex and $\left(P / \Theta, \leq^{\prime}\right)$ a poset. Moreover, from Lemma 3.6 we have that any congruence class of $\Theta$ has a greatest element. Put

$$
Q:=\{x \in P \mid x \text { is the greatest element of }[x] \Theta\} .
$$

Then $(Q, *, 1)$ is a subalgebra of $(P, *, 1)$. Assume $a, b \in Q$. Since $\mathbf{P}$ is a strongly sectionally pseudocomplemented poset and $\Theta$ is strong we have $a, b \leq(a * b) * b$ and $a * b,(a * b) * b \in Q$. This implies

$$
\begin{aligned}
L_{Q}(b) & \subseteq L_{Q}\left(U_{Q}(a, b), a * b\right) \subseteq L_{Q}\left(U_{Q}((a * b) * b), a * b\right)=L_{Q}((a * b) * b, a * b)= \\
& =L((a * b) * b, a * b) \cap Q=L(U(a * b, b),(a * b) * b) \cap Q=L(b) \cap Q=L_{Q}(b) .
\end{aligned}
$$


Note that the first inclusion follows from the fact that $b \leq a * b$ and $b \in L_{Q} U_{Q}(a, b)$. The second inclusion follows from the fact that $L_{Q} U_{Q}(a, b) \subseteq L_{Q} U_{Q}((a * b) * b)$. The first equality follows from $L_{Q} U_{Q}((a * b) * b)=L_{Q}((a * b) * b)$. The second equality follows from the definition of $L_{Q}$. Since $\mathbf{P}$ is sectionally pseudocomplemented we have the next but one equality.

Now, let $c \in Q$ be such that $L_{Q}\left(U_{Q}(a, b), c\right)=L_{Q}(b)$. We have $U_{Q}(a, b)=$ $U(a, b) \cap Q \subseteq U(a, b)$. Hence $L U(a, b) \subseteq L U_{Q}(a, b)=\downarrow\left(L_{Q} U_{Q}(a, b)\right)$. The last equality follows from the fact that $L_{Q} U_{Q}(a, b) \subseteq L U_{Q}(a, b)$ which yields $\downarrow\left(L_{Q} U_{Q}(a, b)\right) \subseteq$ $\downarrow\left(L U_{Q}(a, b)\right)=L U_{Q}(a, b)$ and from the fact that $y \in L U_{Q}(a, b)$ implies $y \leq x \in$ $L_{Q} U_{Q}(a, b)$ where $x$ is the greatest element of $[y] \Theta$.

We obtain $L(b) \subseteq L(U(a, b), c) \subseteq \downarrow\left(L_{Q} U_{Q}(a, b) \cap L_{Q}(c)\right)=\downarrow\left(L_{Q}(b)\right)=L(b)$ since $b, c \in Q$. Since $\mathbf{P}$ is a sectionally pseudocomplemented poset we have $c \leq a * b$, i.e., $(Q, *, 1)$ is sectionally pseudocomplemented. Since $(Q, *, 1)$ is a subalgebra of $(P, *, 1)$ we have that $(Q, *, 1)$ is strongly sectionally pseudocomplemented. Moreover, $x \mapsto[x] \Theta$ an isomorphism from $(Q, \leq, *, 1)$ to $\left(P / \Theta, \leq^{\prime}, *^{\prime},[1] \Theta\right)$ and hence $\left(P / \Theta, \leq^{\prime}, *^{\prime},[1] \Theta\right)$ is also a strongly sectionally pseudocomplemented poset.

The following lemma shows that in a strongly sectionally pseudocomplemented poset $(P, \leq, *, 1)$ where every congruence on $(P, *)$ is convex, all principal congruences $\Theta(a, b)$ with $a \leq b$ are of the form $\Theta(c, 1)$ (i.e. $(P, *)$ has so-called em transferable principal congruences, see [4]).

Lemma 3.9 Let $(P, \leq, *, 1)$ be a strongly sectionally pseudocomplemented poset, assume that every congruence on $(P, *)$ is convex and let $a, b \in P$ with $a \leq b$. Then $\Theta(b * a, 1)=$ $\Theta(a, b)$.

Proof Since $(a, b) \in \Theta(a, b)$ yields $(b * a, 1)=(b * a, b * b) \in \Theta(a, b)$, we have $\Theta(b *$ $a, 1) \subseteq \Theta(a, b)$. Conversely, $(b * a, 1) \in \Theta(b * a, 1)$ yields $((b * a) * a, a)=((b * a) *$ $a, 1 * a) \in \Theta(b * a, 1)$ which because of $a \leq b \leq(b * a) * a$ and the convexity of $\Theta(b * a, 1)$ implies $(a, b) \in \Theta(b * a, 1)$, i.e. $\Theta(a, b) \subseteq \Theta(b * a, 1)$.

\section{Completion of Sectionally Pseudocomplemented Posets}

Now we consider the Dedekind-MacNeille completion of sectionally pseudocomplemented posets.

In contrast to the situation of relatively pseudocomplemented posets the DedekindMacNeille completion of a strongly sectionally pseudocomplemented poset $\mathbf{P}$ need not be sectionally pseudocomplemented, even if $\mathbf{P}$ is finite and has a greatest element.

Example 4.1 Though the poset visualized in Fig. 5 is strongly sectionally pseudocomplemented with the operation table for $*$ as below, its Dedekind-MacNeille completion visualized in Fig. 6 is not sectionally pseudocomplemented since $a * 0$ does not exist.

For our next investigations, we introduce the following useful concepts.

Definition 4.2 Let $(I, \leq)$ be a chain with greatest element $\top$ and smallest element $\perp$. Let $\mathbf{P}_{i}=\left(P_{i}, \leq_{i}\right), i \in I$, be a family of posets such that $\mathbf{P}_{\top}$ has a greatest element 1 and such that the following hold: 


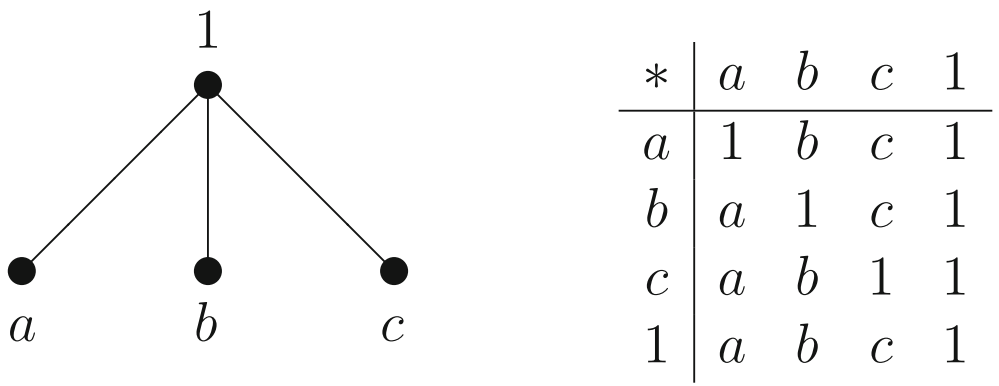

Fig. 5

(i) If $i \in I$, then there are $a, b \in P_{i}$ with $a<b$,

(ii) if $i, j, k \in I$ and $i<k<j$, then $P_{i} \cap P_{j}=\emptyset$,

(iii) if $i, j \in I$ and $i<j$, then $\left|P_{i} \cap P_{j}\right| \leq 1$,

(iv) if $i, j \in I, i<j$ and $P_{i} \cap P_{j}=\{a\}$, then $P_{i}=L_{P_{i}}(a)$ and $P_{j}=U_{P_{j}}(a)$.

Put $P=\bigcup_{i \in I} P_{i}$. For $a, b \in P$, say $a \in P_{i}$ and $b \in P_{j}$ with $i, j \in I$, define

$$
a \leq b \text { if and only if } a=b \text { or }\left(i=j \text { and } a \leq_{i} b\right) \text { or } i<j .
$$

We call $\mathbf{P}=(P, \leq)$ the generalized ordinal sum of $\mathbf{P}_{i}, i \in I$.

It is elementary that $\mathbf{P}$ is a poset with a greatest element 1 .

Now, we can state some sufficient conditions under which the Dedekind-MacNeille completion of a sectionally pseudocomplemented poset is sectionally pseudocomplemented. By [9] the Dedekind-MacNeille completion of a poset $\mathbf{P}$ is (up to isomorphism) any complete

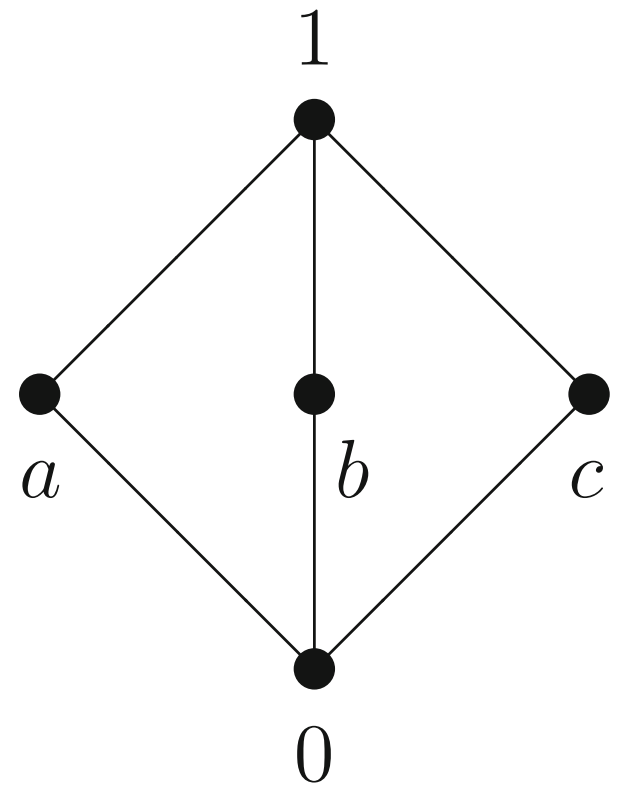

Fig. 6 
lattice $\mathbf{Q}$ into which $\mathbf{P}$ can be supremum-densely and infimum-densely embedded (i.e., for every element $x \in Q$ there exist $M, N \subseteq P$ such that $x=\bigvee \varphi(M)=\bigwedge \varphi(N)$, where $\varphi: P \rightarrow Q$ is the embedding). We usually identify $P$ with $\varphi(P)$. In this sense $\mathbf{Q}$ preserves all infima and suprema existing in $\mathbf{P}$.

Now we turn our attention to a notion of a DM-yoked family of a generalized ordinal sum. The importance of this concept is based on the fact that under natural assumptions (which are e.g. satisfied for a finite index set $I$ ) the Dedekind-MacNeille completion of a generalized ordinal sum will be isomorphic to a generalized ordinal sum of the respective DM-yoked family.

Definition 4.3 Let $\mathbf{P}=(P, \leq)$ be the generalized ordinal sum of $\mathbf{P}_{i}=\left(P_{i}, \leq_{i}\right), i \in I$. We say that a family $\mathbf{Q}_{i}=\left(Q_{i}, \leq_{i}\right), i \in I$, of posets is a DM-yoked family of $\mathbf{P}$ if the following conditions are satisfied:

(y1) $\mathbf{P}_{i}$ is a sub-poset of $\mathbf{Q}_{i}$ such that $\mathbf{Q}_{i}$ is the Dedekind-MacNeille completion of $\mathbf{P}_{i}$ for every $i \in I$,

(y2) if $i, j, k \in I$ and $i<k<j$, then $Q_{i} \cap Q_{j}=\emptyset$,

(y3) if $i, j \in I$ and $i<j$, then $\left|Q_{i} \cap Q_{j}\right| \leq 1$,

(y4) if $i, j \in I, i \prec j, P_{i} \cap P_{j}=\emptyset, \mathbf{P}_{i}$ has a greatest element and $\mathbf{P}_{j}$ has a smallest element, then $Q_{i} \cap Q_{j}=\emptyset$,

(y5) if $i, j \in I, i \prec j, P_{i} \cap P_{j}=\emptyset, \mathbf{P}_{i}$ does not have a greatest element and $\mathbf{P}_{j}$ has a smallest element $0_{P_{j}}$, then $0_{P_{j}}$ is the greatest element of $\mathbf{Q}_{i}$,

(y6) if $i, j \in I, i \prec j, P_{i} \cap P_{j}=\emptyset, \mathbf{P}_{j}$ does not have a smallest element and $\mathbf{P}_{i}$ has a greatest element $1_{P_{i}}$, then $1_{P_{i}}$ is the smallest element of $\mathbf{Q}_{j}$,

(y7) if $i, j \in I, i \prec j, P_{i} \cap P_{j}=\emptyset, \mathbf{P}_{j}$ does not have a smallest element and $\mathbf{P}_{i}$ does not have a greatest element, then the greatest element $1_{Q_{i}}$ of $\mathbf{Q}_{i}$ is the smallest element $0_{Q_{j}}$ of $\mathbf{Q}_{j}$

(y8) if $i, j \in I, i<j$ and $Q_{i} \cap Q_{j}=\{a\}$, then $a$ is the greatest element of $\mathbf{Q}_{i}$ and the smallest element of $\mathbf{Q}_{j}$.

The question when there exists a DM-yoked family for a given poset $\mathbf{P}$ which is a generalized ordinal sum of posets $\mathbf{P}_{i}=\left(P_{i}, \leq_{i}\right), i \in I$, is positively answered in the following series of lemmas.

We will first need the following definition.

Definition 4.4 Let $\mathbf{P}=(P, \leq)$ be the generalized ordinal sum of $\mathbf{P}_{i}=\left(P_{i}, \leq_{i}\right), i \in I$. We say that a family $\mathbf{R}_{i}=\left(R_{i}, \leq_{i}\right), i \in I$, of posets is a DM-related family of $\mathbf{P}$ if the following conditions are satisfied:

(r1) $\mathbf{P}_{i}$ is a sub-poset of $\mathbf{R}_{i}$ such that $\mathbf{R}_{i}$ is the Dedekind-MacNeille completion of $\mathbf{P}_{i}$ for every $i \in I$,

(r2) For every $i \in I, 0_{R_{i}}<1_{R_{i}}$,

(r3) If $i<k<j$, then $R_{i} \cap R_{j}=\emptyset$,

(r4) For $i \prec j, R_{i} \cap R_{j} \neq \emptyset$ if and only if $R_{i} \cap R_{j}=\{a\}$ with $1_{R_{i}}=a=0_{R_{j}}$.

Let $\mathbf{P}=(P, \leq)$ be the generalized ordinal sum of $\mathbf{P}_{i}=\left(P_{i}, \leq_{i}\right), i \in I$. For every $i \in I$, let $\mathbf{R}_{i}=\left(R_{i}, \leq_{i}\right)$ be the Dedekind-MacNeille completion of $\mathbf{P}_{i}$. We can assume without loss of generality that

(a) $\quad R_{i} \cap R_{j}=\emptyset$ if and only if $P_{i} \cap P_{j}=\emptyset$, and 
(b) $\quad R_{i} \cap R_{j}=\{a\}$ if and only if $P_{i} \cap P_{j}=\{a\}$.

Hence a DM-related family $\mathbf{R}_{i}=\left(R_{i}, \leq_{i}\right), i \in I$, of $\mathbf{P}$ always exists.

Lemma 4.5 Let $\mathbf{P}=(P, \leq)$ be the generalized ordinal sum of $\mathbf{P}_{i}=\left(P_{i}, \leq_{i}\right), i \in I$. Then a DM-yoked family $\mathbf{Q}_{i}=\left(Q_{i}, \leq_{i}\right), i \in I$, of $\mathbf{P}$ exists.

Proof Let $\mathbf{R}_{i}=\left(R_{i}, \leq_{i}\right), i \in I$, be the DM-related family of $\mathbf{P}$ which always exists. We will proceed in two steps.

Step 1: Let $i \in I$. Assume that there exists some $j \in I$ with $i \prec j$ and $P_{i} \cap P_{j}=\emptyset$. If $\mathbf{P}_{i}$ does not have a greatest element we put $S_{i}:=\left(R_{i} \backslash\left\{1_{R_{i}}\right\}\right) \cup\left\{0_{R_{j}}\right\}$ such that $0_{R_{j}}$ will be the greatest element of $S_{i}$ and the order $\leq_{i}$ on $S_{i}$ restricted to $R_{i} \backslash\left\{1_{R_{i}}\right\}$ will be the restriction of the order on $\mathbf{R}_{i}$. Clearly $P_{i} \subseteq S_{i}$ and $\mathbf{P}_{i}$ is a sub-poset of $\mathbf{S}_{i}$. If $\mathbf{P}_{i}$ does have a greatest element, we put $\mathbf{S}_{i}:=\mathbf{R}_{i}$. If $P_{i} \cap P_{j}=\{a\}$, then $a=1_{P_{i}}=0_{P_{j}}$. Hence also $a=1_{R_{i}}=0_{R_{j}}$ and we put $\mathbf{S}_{i}:=\mathbf{R}_{i}$. If there is no $j \in I$ such that $i \prec j$, we put again $\mathbf{S}_{i}:=\mathbf{R}_{i}$.

Let us verify that $\mathbf{S}_{i}=\left(S_{i}, \leq S_{i}\right), i \in I$, is a DM-related family of $\mathbf{P}$. Since every $\mathbf{S}_{i}$ is isomorphic to $\mathbf{R}_{i}$, conditions (r1) and (r2) are satisfied.

Let us check condition (r3). Assume $i<k<j$ and $a \in S_{i} \cap S_{j}$. Since $R_{i} \cap R_{j}=\emptyset$ we have $a \notin R_{i}$ or $a \notin R_{j}$. Suppose first that $a \notin R_{i}$. Then the poset $\mathbf{P}_{i}$ does not have a greatest element, there exists some $l \in I$ with $i \prec l \leq k<j, a=0_{R_{l}}<1_{R_{l}}$ and $P_{i} \cap P_{l}=\emptyset$. Since $l<j, a=0_{R_{l}} \notin R_{j}$. Hence $a \in S_{j} \backslash R_{j}$, i.e., $a=0_{R_{m}}$ for some $m \in I, j \prec m$. We have $l<j<m$ and $a \in R_{l} \cap R_{m}$, a contradiction with (r3) for the family $\mathbf{R}_{i}, i \in I$. Second, assume that $a \notin R_{j}$. Therefore $a=0_{R_{m}}$ for some $m \in I, j \prec m$. Since $i<k<j<m$ we have $R_{i} \cap R_{m}=\emptyset$. Thus $a \notin R_{i}$, i.e., $a=0_{R_{l}}$ for some $l \in I, i \prec l \leq k$. Hence $a \in R_{l} \cap R_{m}$ and $l<j<m$, again a contradiction with (r3) for the family $\mathbf{R}_{i}, i \in I$.

Let us see that $\mathbf{S}_{i}, i \in I$ satisfies, condition (r4). Assume that $i \prec j, a \in S_{i} \cap S_{j}$.

Case 1: $S_{i}=R_{i}$ and $S_{j}=R_{j}$. Then $a=1_{R_{i}}=1_{S_{i}}$ and $a=0_{R_{j}}=0_{S_{j}}$.

Case 2: $S_{i}=R_{i}$ and $S_{j}=\left(R_{j} \backslash\left\{1_{R_{j}}\right\}\right) \cup\left\{0_{R_{k}}\right\}$ for $k \in I, j \prec k$. Hence

$$
a \in S_{i} \cap S_{j}=R_{i} \cap\left[\left(R_{j} \backslash\left\{1_{R_{j}}\right\}\right) \cup\left\{0_{R_{k}}\right\}\right]=R_{i} \cap R_{j} .
$$

Thus $R_{i} \cap R_{j}=\{a\}$ with $a=1_{R_{i}}=1_{S_{i}}$ and $a=0_{R_{j}}=0_{S_{j}}$.

Case 3: $S_{i}=\left(R_{i} \backslash\left\{1_{R_{i}}\right\}\right) \cup\left\{0_{R_{j}}\right\}$ and $S_{j}=R_{j}$. We have

$$
a \in S_{i} \cap S_{j}=R_{j} \cap\left[\left(R_{i} \backslash\left\{1_{R_{i}}\right\}\right) \cup\left\{0_{R_{j}}\right\}\right]=\left\{0_{R_{j}}\right\} .
$$

Hence $a=0_{R_{j}}=0_{S_{j}}$ and $a=1_{S_{i}}$.

Case 4: $S_{i}=\left(R_{i} \backslash\left\{1_{R_{i}}\right\}\right) \cup\left\{0_{R_{j}}\right\}$ and $S_{j}=\left(R_{j} \backslash\left\{1_{R_{j}}\right\}\right) \cup\left\{0_{R_{k}}\right\}$ for $k \in I, j \prec k$. Then

$$
S_{i} \cap S_{j}=\left[\left(R_{i} \backslash\left\{1_{R_{i}}\right\}\right) \cup\left\{0_{R_{j}}\right\}\right] \cap\left[\left(R_{j} \backslash\left\{1_{R_{j}}\right\}\right) \cup\left\{0_{R_{k}}\right\}\right]=\left\{0_{R_{j}}\right\} \cap\left(R_{j} \backslash\left\{1_{R_{j}}\right\}\right)=\left\{0_{R_{j}}\right\} .
$$

We obtain that $a=0_{R_{j}}=1_{S_{i}}=0_{S_{j}}$.

Step 2: Let $j \in I$. Assume there exists some $i \in I$ with $i \prec j$ and $P_{i} \cap P_{j}=\emptyset$. If $\mathbf{P}_{j}$ does not have a smallest element, we put $Q_{j}:=\left(S_{j} \backslash\left\{0_{S_{j}}\right\}\right) \cup\left\{1_{S_{i}}\right\}$ such that $1_{S_{i}}$ will be the smallest element of $Q_{j}$ and the order $\leq_{j}$ on $Q_{j}$ restricted to $S_{j} \backslash\left\{0_{S_{j}}\right\}$ will be the restriction of the order on $\mathbf{S}_{j}$. Clearly $P_{j} \subseteq Q_{j}$ and $\mathbf{P}_{j}$ is a sub-poset of $\mathbf{Q}_{j}$. Otherwise we always put $\mathbf{Q}_{j}:=\mathbf{S}_{j}$.

Since $\mathbf{S}_{i}=\left(S_{i}, \leq S_{i}\right), i \in I$, is a DM-related family of $\mathbf{P}$ by the same reasonings as above we can show that $\mathbf{Q}_{i}=\left(Q_{i}, \leq Q_{i}\right), i \in I$, is a DM-related family of $\mathbf{P}$. 
Let us now check that $\mathbf{Q}_{i}=\left(Q_{i}, \leq Q_{i}\right), i \in I$, is a DM-yoked family of $\mathbf{P}$. (y1), (y2): This follows immediately from (r1) and (r3).

(y3), (y8): This follows immediately from (r3) and (r4).

(y4) Assume $i, j \in I, i \prec j, P_{i} \cap P_{j}=\emptyset, \mathbf{P}_{i}$ has a greatest element $1_{P_{i}}$ and $\mathbf{P}_{j}$ has a smallest element $0_{P_{j}}$. Then $R_{i} \cap R_{j}=\emptyset, S_{i}=R_{i}$ and $Q_{j}=S_{j}$. Assume $a \in$ $Q_{i} \cap Q_{j}$. Then $a \in Q_{i} \cap S_{j}$. We have either $a=1_{S_{h}}$ for some $h \in I$ with $h \prec i$ or $a \in S_{i}=R_{i}$, and either $a \in R_{j}$ or $a=0_{R_{k}}$ for some $k \in I$ with $j \prec k$. We can assume

$a \in S_{i}=R_{i}$ or $a \in R_{h} \cup R_{i}$ for some $h \prec i<j$ and $a \in S_{j} \subseteq R_{j} \cup R_{k}$. Moreover, $R_{h} \cap\left(R_{j} \cup R_{k}\right)=\emptyset$ and $R_{i} \cap\left(R_{j} \cup R_{k}\right)=\emptyset$, a contradiction. We conclude $Q_{i} \cap Q_{j}=\emptyset$.

(y5) Suppose $i, j \in I, i \prec j, P_{i} \cap P_{j}=\emptyset, \mathbf{P}_{i}$ does not have a greatest element and $\mathbf{P}_{j}$ has a smallest element $0_{P_{j}}$. We have $R_{i} \cap R_{j}=\emptyset, Q_{j}=S_{j}$ and $S_{i}=\left\{0_{R_{j}}\right\} \cup\left(R_{i} \backslash\left\{1_{R_{i}}\right\}\right)$. Clearly, $0_{R_{j}} \in Q_{i}$ and $0_{R_{j}} \in S_{j}=Q_{j}$. Hence $0_{P_{j}}=0_{R_{j}} \in Q_{i} \cap Q_{j}$ is the greatest element of $\mathbf{Q}_{i}$.

(y6) Assume $i, j \in I, i \prec j, P_{i} \cap P_{j}=\emptyset, \mathbf{P}_{j}$ does not have a smallest element and $\mathbf{P}_{i}$ has a greatest element $1_{P_{i}}$. We obtain $S_{i}=R_{i}, Q_{j}=\left(S_{j} \backslash\left\{0_{S_{j}}\right\}\right) \cup\left\{1_{S_{i}}\right\}$ and $R_{i} \cap R_{j}=\emptyset$. We have $1_{P_{i}}=1_{R_{i}}=1_{S_{i}} \in Q_{j}$ and $1_{S_{i}} \in Q_{i}$. Hence $1_{P_{i}}$ is the smallest element of $\mathbf{Q}_{j}$.

(y7) Assume $i, j \in I, i \prec j, P_{i} \cap P_{j}=\emptyset, \mathbf{P}_{j}$ does not have a smallest element and $\mathbf{P}_{i}$ does not have a greatest element. Then $S_{i}=\left\{0_{R_{j}}\right\} \cup\left(R_{i} \backslash\left\{1_{R_{i}}\right\}\right)$ and $Q_{j}=$ $\left(S_{j} \backslash\left\{0_{S_{j}}\right\}\right) \cup\left\{1_{S_{i}}\right\}$. Since $1_{S_{i}}=0_{R_{j}}=0_{Q_{j}}$ and $1_{S_{i}}=1_{Q_{i}}$, we obtain that the greatest element $1_{Q_{i}}$ of $\mathbf{Q}_{i}$ is the smallest element $0_{Q_{j}}$ of $\mathbf{Q}_{j}$.

Finally, we can state our results on Dedekind-MacNeille completion of posets which are the generalized ordered sum of their parts $\left(P_{i}, \leq_{i}\right), i \in I$.

Theorem 4.6 Let $\mathbf{P}=(P, \leq)$ be the generalized ordinal sum of $\mathbf{P}_{i}=\left(P_{i}, \leq_{i}\right), i \in I$, and $\mathbf{Q}_{i}=\left(Q_{i}, \leq_{i}\right), i \in I$, be a DM-yoked family of $\mathbf{P}$. Then the generalized ordinal sum $\mathbf{Q}=(Q, \leq)$ of $\mathbf{Q}_{i}=\left(Q_{i}, \subseteq\right), i \in I$, exists. If any non-empty subset of I has a maximal element, then $\mathbf{D M}(\mathbf{P}) \cong \mathbf{Q}$.

Proof First let us check that the assumptions of Definition 4.2 are satisfied. Clearly, $\mathbf{Q}_{i}, i \in I$, is a family of posets such that $\mathbf{Q}_{\top}$ has a greatest element 1 (we may identify 1 with $1_{P_{\top}}$ ) and condition (i) is satisfied by (y1). Condition (ii) follows from (y2) and condition (iii) from (y3). From (y8) we obtain condition (iv). Hence the generalized ordinal sum $\mathbf{Q}=(Q, \leq)$ of $\mathbf{Q}_{i}=\left(Q_{i}, \subseteq\right), i \in I$, exists.

Assume now that any non-empty subset of $I$ has a maximal element and $I$ has a smallest element $\perp$. Let us show $\mathbf{Q} \cong \mathbf{D M}(\mathbf{P})$. Note that we have, for every $i \in I$, order isomorphisms $\varphi_{i}: Q_{i} \rightarrow \operatorname{DM}\left(P_{i}\right)$ and $\psi_{i}: \operatorname{DM}\left(P_{i}\right) \rightarrow Q_{i}$ defined by $\varphi_{i}(a):=L_{P_{i}} U_{P_{i}}\left(\left\{x \in P_{i} \mid x \leq\right.\right.$ $a\})$ and $\psi_{i}(B):=\bigvee_{Q_{i}}\left(B \cap P_{i}\right)$ for all $a \in Q_{i}$ and $B \in \operatorname{DM}\left(P_{i}\right)$, and $\varphi_{i} \circ \psi_{i}=\operatorname{id}_{\mathrm{DM}\left(P_{i}\right)}$, $\psi_{i} \circ \varphi_{i}=\operatorname{id}_{Q_{i}}$.

We define mappings $\varphi: Q \rightarrow \operatorname{DM}(P)$ and $\psi: \operatorname{DM}(P) \rightarrow Q$ as follows:

$$
\varphi(a):=L U(\{x \in P \mid x \leq a\}) \text { and } \psi(B):= \begin{cases}\psi_{\perp}(\emptyset) & \text { if } B=\emptyset \\ \bigvee_{Q_{j}}\left(B \cap P_{j}\right) & \text { otherwise }\end{cases}
$$


where $j:=\max _{B \cap P_{m} \neq \emptyset} m(a \in Q, B \in \mathrm{DM}(P))$. Clearly, $\varphi$ and $\psi$ are well-defined and order-preserving. Recall also that $\varphi(a)=\varphi_{k}(a) \cup \bigcup_{m<k} P_{m}$ where $k:=\max _{a \in Q_{m}} m$. We have

$$
\psi(\varphi(a))=\psi\left(\varphi_{k}(a) \cup \bigcup_{m<k} P_{m}\right)=\bigvee_{Q_{k}}\left(\left(\varphi_{k}(a) \cup \bigcup_{m<k} P_{m}\right) \cap P_{k}\right)=\psi_{k}\left(\varphi_{k}(a)\right)=a
$$

for all $a \in Q$, here $k:=\max _{a \in Q_{m}} m$. Let $B \in \mathrm{DM}(P)$. If $B=\varnothing$ and $B=L U(B)$, then $\varphi(\psi(\emptyset))=\varphi\left(\psi_{\perp}(\emptyset)\right)=\varphi_{\perp}\left(0_{Q_{\perp}}\right)$. Assume $x \in \varphi_{\perp}\left(0_{Q_{\perp}}\right)$. Then $x$ is the smallest element of $\mathbf{P}_{\perp}$, i.e., $x \leq p$ for all $p \in P$, i.e., $x \in B$, a contradiction. Hence $\varphi(\psi(\emptyset))=\emptyset$. Suppose now that $B \neq \emptyset$ and put $j:=\max _{B \cap P_{m} \neq \emptyset} m$. Then

$$
\varphi(\psi(B))=\varphi\left(\bigvee_{Q_{j}}\left(B \cap P_{j}\right)\right)=\varphi_{j}\left(\bigvee_{Q_{j}}\left(B \cap P_{j}\right)\right) \cup \bigcup_{m<j} P_{m}=\left(B \cap P_{j}\right) \cup \bigcup_{m<j} P_{m}=B .
$$

Now we show that the construction of a generalized ordinal sum preserves the property of sectional pseudocomplementation.

Theorem 4.7 Let $\mathbf{P}=(P, \leq)$ be a generalized ordinal sum of $\mathbf{P}_{i}=\left(P_{i}, \leq_{i}\right), i \in I$, assume that $\left(P_{i}, \leq, *_{i}\right)$ are sectionally pseudocomplemented for all $i \in I$, that $j \in I, L_{j}\left(P_{j}\right)=\emptyset$ implies $U_{s}\left(P_{s}\right)=\emptyset$ where $s:=\max _{m<j} m$ and that any non-empty subset of I has a maximal element. Then $\mathbf{P}$ is sectionally pseudocomplemented.

Proof Let $i, j \in I, a \in P_{i}$ and $b \in P_{j}$ such that $i$ and $j$ are maximal with this property. We put

$$
a * b:= \begin{cases}1 & \text { if } a \leq b, \\ a *_{i} b & \text { if } a \not \leq b \text { and } i=j, \\ b & \text { if } a \not \leq b \text { and } i>j .\end{cases}
$$

We prove that $a * b$ is the sectional pseudocomplement of $a$ and $b$ in $\mathbf{P}$.

Case 1. $a \leq b$.

We have $L(U(a, b), 1)=L(b, 1)=L(b)$.

Case 2. $a \not \leq b$ and $i=j$.

We have

$$
\begin{aligned}
L\left(U(a, b), a *_{i} b\right) & =L\left(U(a, b) \cap P_{i}, a *_{i} b\right)=\left(L\left(U(a, b) \cap P_{i}, a *_{i} b\right) \cap P_{i}\right) \cup \bigcup_{m<i} P_{m}= \\
& =L_{i}\left(U_{i}(a, b), a *_{i} b\right) \cup \bigcup_{m<i} P_{m}=L_{i}(b) \cup \bigcup_{m<i} P_{m}=L(b) .
\end{aligned}
$$

Now assume $L(U(a, b), c)=L(b), c \in P_{k}, k \in I$. Then $b \leq c$ which implies $k \geq i$. Now $k>i$ would imply

$$
a \in L(U(a, b), c)=L(b),
$$

a contradiction. Hence $k=i$ and

$$
L_{i}\left(U_{i}(a, b), c\right)=L(U(a, b), c) \cap P_{i}=L(b) \cap P_{i}=L_{i}(b)
$$

which implies $c \leq a *_{i} b$.

Case 3. $a \not \leq b$ and $i>j$.

We have $L(U(a, b), b)=L(b)$. Now assume $L(U(a, b), c)=L(b), c \in P_{k}, k \in I$. Then 
$b \leq c$ which implies $k \geq j$ and $L(b)=L(a, c)$.

Case 3a. $i>k$.

We have $c \leq b$ which yields $b=c$.

Case 3b. $i<k$.

We have $L(b)=L(a, c)=L(a)$, i.e., $a \leq b$, a contradiction.

Case 3c. $i=k$.

We obtain $k>j$. Since $L(b)=L(a, c), b=1_{j}$ is the greatest element of $\mathbf{P}_{j}$. If $\mathbf{P}_{i}$ has a smallest element $0_{i}$, then necessarily $b=0_{i} \in P_{i}$, a contradiction to the assumption that $j$ is the maximal index from $I$ with $b \in P_{j}$. Hence $\mathbf{P}_{i}$ does not have a smallest element. Put $r:=\max _{m<k} m$. Assume first that $j<r<k=i$. Then there exist by Definition 4.2 (i) elements $x, y \in P_{r}$ with $b \leq x<y \leq a, c$. We conclude $y \in L(a, c), y \not \leq b$, a contradiction. Suppose now that $j=r<k=i$. Since $L_{i}\left(P_{i}\right)=\emptyset$ and $b$ is the greatest element of $\mathbf{P}_{j}$ we obtain $\{b\}=U_{r}\left(P_{r}\right)=\emptyset$, a contradiction. Hence the only possible case is 3 a which yields $b=c$ and finally that $\mathbf{P}$ is sectionally pseudocomplemented.

Altogether, we can summarize our results as follows.

Corollary 4.8 Let $\mathbf{P}=(P, \leq)$ be the generalized ordinal sum of $\mathbf{P}_{i}=\left(P_{i}, \leq_{i}\right), i \in I$. Let $\left(\operatorname{DM}\left(\mathbf{P}_{i}\right), \leq_{i}, *_{i}\right)$ be sectionally pseudocomplemented for all $i \in I$ and assume that any non-empty subset of $I$ has a maximal element and that $j \in I$ and $L_{j}\left(P_{j}\right)=\emptyset$ imply $U_{s}\left(P_{s}\right)=\emptyset$ where $s:=\max _{m<j} m$. Then $\mathbf{D M}(\mathbf{P})$ is sectionally pseudocomplemented.

Proof From Lemma 4.5 we have that there exists a DM-yoked family $\mathbf{Q}_{i}=\left(Q_{i}, \leq_{i}\right)$, $i \in I$, of $\mathbf{P}$ such that $\mathbf{Q}_{i}$ is isomorphic to a sectionally pseudocomplemented poset $\mathbf{D M}\left(\mathbf{P}_{i}\right)$ for every $i \in I$. From Theorem 4.6 we know that $\mathbf{D M}(\mathbf{P})$ is isomorphic to the generalized ordinal sum $\mathbf{Q}$ of the DM-yoked family $\mathbf{Q}_{i}=\left(Q_{i}, \leq_{i}\right), i \in I$, of $\mathbf{P}$. Since every $\mathbf{Q}_{i}$ is sectionally pseudocomplemented we have from Theorem 4.7 that $\mathbf{Q}$ and hence also $\mathbf{D M}(\mathbf{P})$ are sectionally pseudocomplemented.

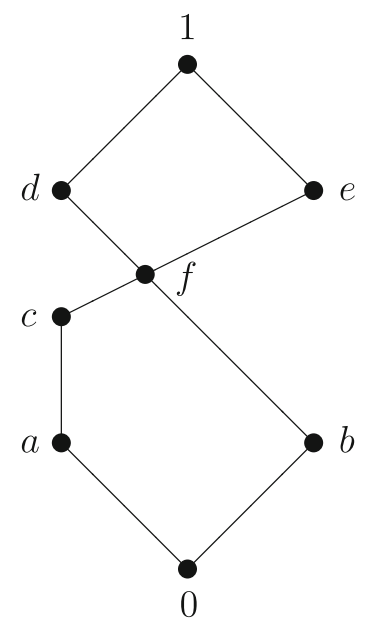

\begin{tabular}{c|cccccccc}
$*$ & 0 & $a$ & $b$ & $c$ & $d$ & $e$ & $f$ & 1 \\
\hline 0 & 1 & 1 & 1 & 1 & 1 & 1 & 1 & 1 \\
$a$ & $b$ & 1 & $b$ & 1 & 1 & 1 & 1 & 1 \\
$b$ & $c$ & $a$ & 1 & $c$ & 1 & 1 & 1 & 1 \\
$c$ & $b$ & $a$ & $b$ & 1 & 1 & 1 & 1 & 1 \\
$d$ & 0 & $a$ & $b$ & $c$ & 1 & $e$ & $e$ & 1 \\
$e$ & 0 & $a$ & $b$ & $c$ & $d$ & 1 & $d$ & 1 \\
$f$ & 0 & $a$ & $b$ & $c$ & 1 & 1 & 1 & 1 \\
1 & 0 & $a$ & $b$ & $c$ & $d$ & $e$ & $f$ & 1
\end{tabular}

Fig. 7 
The situation described in Theorem 4.7 and Corollary 4.8 can be illustrated by the following example.

Example 4.9 Consider the sectionally pseudocomplemented poset $\mathbf{P}$ visualized in Fig. 2. It is evident that $\mathbf{P}$ is the generalized ordinal sum of the sectionally pseudocomplemented posets $\mathbf{P}_{1}=\left(P_{1}, \leq\right)=(\{0, a, b, c\}, \leq)$ and $\mathbf{P}_{2}=\left(P_{2}, \leq\right)=(\{d, e, 1\}, \leq)$. Of course, $P_{1} \cap P_{2}=\emptyset$. Hence the conditions of Definition 4.2 are satisfied. Then $\mathbf{D M}\left(\mathbf{P}_{1}\right)$ is the lattice $\mathrm{N}_{5}$ and $\mathbf{D M}\left(\mathbf{P}_{2}\right)$ the four-element Boolean algebra, i.e. both are sectionally pseudocomplemented lattices. The generalized ordinal sum of $\mathbf{D M}\left(\mathbf{P}_{1}\right)$ and $\mathbf{D M}\left(\mathbf{P}_{2}\right)$ is visualized in Fig. 7. According to Theorem 4.7 it is again sectionally pseudocomplemented.

The Dedekind-MacNeille completion of the poset visualized in Fig. 3 is visualized in Fig. 7. Here $L(x)$ is abbreviated by $x$ for $x \in\{0, a, b, c, d, e, 1\}$ and $f$ is an abbreviation of $L(d, e)$. The operation table of $*$ in $\mathbf{D M}(\mathbf{P})$ is shown in Fig. 7.

According to Corollary 4.8, $\mathbf{D M}(\mathbf{P})$ is just the generalized ordinal sum of $\mathbf{D M}\left(\mathbf{P}_{1}\right)$ and $\operatorname{DM}\left(\mathbf{P}_{2}\right)$.

Acknowledgments The authors would like to express their gratitude to the anonymous reviewer whose thorough and detailed comments led to a considerable improvement of this paper.

Funding Open access funding provided by TU Wien (TUW).

Data availability statement No datasets were generated or analyzed during the current study.

Open Access This article is licensed under a Creative Commons Attribution 4.0 International License, which permits use, sharing, adaptation, distribution and reproduction in any medium or format, as long as you give appropriate credit to the original author(s) and the source, provide a link to the Creative Commons licence, and indicate if changes were made. The images or other third party material in this article are included in the article's Creative Commons licence, unless indicated otherwise in a credit line to the material. If material is not included in the article's Creative Commons licence and your intended use is not permitted by statutory regulation or exceeds the permitted use, you will need to obtain permission directly from the copyright holder. To view a copy of this licence, visit http://creativecommonshorg/licenses/by/4.0/.

\section{References}

1. Balbes, R.: On free pseudo-complemented and relatively pseudo-complemented semi-lattices. Fund. Math. 78, 119-131 (1973)

2. Birkhoff, G.: Lattice Theory. Amer. Math. Soc., Providence, R. I. ISBN 0-8218-1025-1 (1979)

3. Chajda, I.: An extension of relative pseudocomplementation to non-distributive lattices. Acta Sci. Math. (Szeged) 69, 491-496 (2003)

4. Chajda, I., Eigenthaler, G., Länger, H.: Congruence Classes in Universal Algebra. Heldermann, Lemgo. ISBN 3-88538-226-1 (2012)

5. Chajda, I., Kühr, J., Länger, H.: Relatively residuated lattices and posets. Math. Slovaca 70, 239-250 (2020)

6. Chajda, I., Radeleczki, S.: On varieties defined by pseudocomplemented nondistributive lattices. Publ. Math. Debrecen 63, 737-750 (2003)

7. Dilworth, R.P.: Non-commutative residuated lattices. Trans. Amer. Math. Soc. 46, $426-444$ (1939)

8. Pawar, Y.S.: Implicative posets. Bull. Calcutta Math. Soc. 85, 381-384 (1993)

9. Schmidt, J.: Zur Kennzeichnung der Dedekind-MacNeilleschen Hülle einer geordneten Hülle. Arch. Math. (Basel) 7, 241-249 (1956)

10. Venkatanarasimhan, P.V.: Pseudo-complements in posets. Proc. Amer. Math. Soc. 28, 9-17 (1971) 
Publisher's Note Springer Nature remains neutral with regard to jurisdictional claims in published maps and institutional affiliations.

\section{Affiliations}

\section{Ivan Chajda ${ }^{1} \cdot$ Helmut Länger ${ }^{1,2}$ (D) . Jan Paseka ${ }^{3}$}

Ivan Chajda

ivan.chajda@upol.cz

Jan Paseka

paseka@math.muni.cz

1 Faculty of Science, Department of Algebra and Geometry, Palacký University Olomouc, 17. listopadu 12, 77146 Olomouc, Czech Republic

2 Faculty of Mathematics and Geoinformation, Institute of Discrete Mathematics and Geometry, TU Wien, Wiedner Hauptstraße 8-10, 1040 Vienna, Austria

3 Faculty of Science, Department of Mathematics and Statistics, Masaryk University Brno, Kotlářská 2, 61137 Brno, Czech Republic 